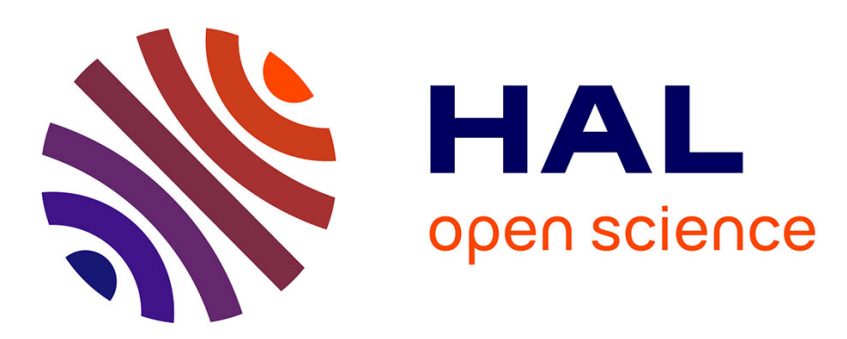

\title{
A Comparison of the Transfer Functions and Flow Fields of Flames With Increasing Swirl Number
}

M. Gatti, R Gaudron, Clement Mirat, Laurent Zimmer, Thierry Schuller

\section{To cite this version:}

M. Gatti, R Gaudron, Clement Mirat, Laurent Zimmer, Thierry Schuller. A Comparison of the Transfer Functions and Flow Fields of Flames With Increasing Swirl Number. ASME Turbo Expo 2018, Jun 2018, Oslo, Norway. 10.1115/gt2018-76105 . hal-01877585

\section{HAL Id: hal-01877585 \\ https://hal.science/hal-01877585}

Submitted on 20 Sep 2018

HAL is a multi-disciplinary open access archive for the deposit and dissemination of scientific research documents, whether they are published or not. The documents may come from teaching and research institutions in France or abroad, or from public or private research centers.
L'archive ouverte pluridisciplinaire HAL, est destinée au dépôt et à la diffusion de documents scientifiques de niveau recherche, publiés ou non, émanant des établissements d'enseignement et de recherche français ou étrangers, des laboratoires publics ou privés. 


\title{
A COMPARISON OF THE TRANSFER FUNCTIONS AND FLOW FIELDS OF FLAMES WITH INCREASING SWIRL NUMBER
}

\author{
M. Gatti ${ }^{1}$, R. Gaudron ${ }^{1}$, C. Mirat ${ }^{1}$, L.Zimmer ${ }^{1}$, T. Schuller ${ }^{1,2}$ \\ ${ }^{1}$ Laboratoire EM2C, CNRS, CentraleSupélec, Université Paris-Saclay, 3, rue Joliot Curie, 91192 Gif-sur-Yvette cedex, France \\ ${ }^{2}$ Institut de Mécanique des Fluides de Toulouse (IMFT), Université de Toulouse, CNRS, INPT, UPS, Toulouse, France \\ Email: marco.gatti@centralesupelec.fr
}

\section{ABSTRACT}

The frequency response of premixed swirled flames is investigated by comparing their Transfer Function (FTF) between velocity and heat release rate fluctuations. The equivalence ratio and flow velocity are kept constant and four different swirling injectors are tested with increasing swirl numbers. The first injector features a vanishing low swirl number $S=0.20$ and produces a flame anchored by the recirculating flow in the wake of a central bluff body. The three other swirling injectors produce highly swirled flows $(S>0.6)$ leading to a much larger internal recirculation region, which size increases with the swirl level. When operating the burner at $S=0.20$, the FTF gain curve smoothly increases to reach a maximum and then smoothly decreases towards zero. For the highly swirled flames $(S>0.6)$, the FTF gain curve shows a succession of valleys and peaks attributed to interferences between axial and azimuthal velocity fluctuations at the injector outlet. The FTF phase-lag curves from the vanishing low and highly swirled flames are the same at low frequencies despite their large differences in flame length and flame aspect ratio. Deviations between the FTF phase lag curves of the different swirled flames start above the frequency corresponding to the first valley in the FTF gain of the highly swirled flames. Phase averaged images of the axial flow fields and of the flame chemiluminescence are used to interpret these features. At forcing frequencies corresponding to peak FTF gain values, the cold flow response of all flames investigated is dominated by large coherent vortical structures shed from the injector lip. At forcing frequencies corresponding to a valley in the FTF gain curve of the highly swirled flames, the formation of large coherent structures is strongly hindered in the cold flow response. These observations contrast with previous interpretations of the mechanisms associated to the low FTF response of swirled flames. It is finally found that for flames stabilized with a large swirl number, heat release rate fluctuations result both from large flame luminosity oscillations and large flame volume oscillations. For conditions leading to a small FTF gain value, both the flame luminosity and flame volume fluctuations are suppressed confirming the $a b$ sence of strong perturbations within the flow at these frequencies. The experiments made in this work reveal a purely hydrodynamic mechanism at the origin of the low response of swirling flames at certain specific frequencies.

\section{INTRODUCTION}

Lean premixed systems powered by swirling flames are inconveniently prone to develop thermoacoustic instabilities [1-3]. The dynamics of these systems is complicated by the complex interactions taking place between the swirling flow, the swirling flame and acoustics [4-6]. One of the main issue is to determine how heat release rate fluctuations $\dot{Q}^{\prime}$ are produced by incoming velocity perturbations $u^{\prime}$, a knowledge necessary if one wishes to understand and eventually control the onset of instabilities.

It is known that the response of the swirler needs to be taken into account [6,7]. Straub and Richards [8] firstly described the influence of the swirler position on self-sustained combustion instabilities in a gas turbine combustor. Komarek and Polifke [9] and later Kim and Santavicca [10] observed a strong dependence of the Flame Transfer Function (FTF) upon the distance from the swirler to the injector outlet. It has been shown that an acoustic pulsation impinging on a swirler generates a vorticity wave, convected downstream at the local flow velocity $[9,11]$. This in turn leads to interferences between axial acoustic and azimuthal convective disturbances and to the peculiar response of swirling flames to acoustic excitations, characterized by alternating FTF gain peaks and valleys as a function of frequency $[9,11,12]$.

In a series of work, Palies and co-workers $[11,13,14]$ found that (i) the same mechanisms take place for radial or axial swirlers, (ii) the frequencies at which the lowest FTF gain val- 
ues are observed are associated to the largest oscillations of the swirl number when azimuthal and axial flow disturbances are out of phase at the injector outlet and (iii) in this latter case, flame vortex roll-up taking place at the flame tip and oscillations of the flame angle at the flame base lead to out of phase contributions to heat release oscillations resulting in a globally weak flame response to the acoustic perturbation.

Effects of the swirl level on the FTF of premixed swirling flames has recently been investigated by Gatti et al. [15]. It has been found that it is possible to lower the minimum value of the FTF gain by increasing the swirl number. This technique can be used to drastically reduce the FTF gain at a fixed forcing frequency up to a limit where flashback takes place within the injector [16]. The mechanism by which this reduction of the FTF gain is achieved slightly differs from the one identified in [14]. In an analysis based on phase conditioned images of the flame dynamics, Gatti et al. [15] could not identify the compensation mechanism described in [14] associated to out of phase responses of the heat release rate produced at the flame tip versus that resulting from the flame base dynamics. The lowest responses of swirled flames were found in [15] to be due to the absence of heat release fluctuations all along the flame.

These observations are used as the basis to further investigate the dynamics of swirling flames. The origin of the physical mechanisms leading to a drop of the FTF gain curve is questioned with a series of experiments conducted under cold and hot flow conditions, for different geometrical configurations of the swirling injector. The observations made are confronted to current interpretations of the response of swirling flames associated with the combined effects of swirl number oscillations and flame vortex roll-up.

The experimental setup and diagnostics used in this work are presented in the next section, followed by a description of the unperturbed flow fields and flames obtained with the different injectors tested. The measured FTF are described and analyzed next. The mechanism at the origin of the peculiar shape of the FTF of swirling flames, both in terms of gain and phase-lag, is then questioned, by phase conditioned analysis of particle image velocimetry measurements made under cold flow conditions. In the last section, the forced flame dynamics is investigated by phase conditioned analysis of the $\mathrm{OH}^{*}$ signals.

\section{EXPERIMENTAL SETUP AND DIAGNOSTICS}

The experimental setup is sketched in Fig. 1. It consists of a plenum, an injection unit, a combustion chamber and a small converging exhaust. A mixture of methane and air, at a fixed equivalence ratio $\phi=0.82$, is injected from two diametrically opposed apertures at the plenum base. The flow crosses a grid and a honeycomb structure to break the largest turbulent scales. A nozzle produces a top-hat velocity profile in a section of $D=22 \mathrm{~mm}$ diameter. The bulk velocity $U_{b}=5.44 \mathrm{~m} / \mathrm{s}$ in this section at
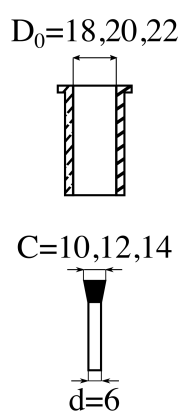

Swirler cut S-S

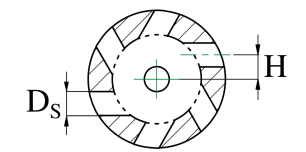

\begin{tabular}{|c|c|c|c|c|}
\hline & $\mathrm{SW}_{1}$ & $\mathrm{SW}_{2}$ & $\mathrm{SW}_{3}$ & $\mathrm{SW}_{4}$ \\
\hline $\begin{array}{c}\mathrm{D}_{\mathrm{S}} \\
{[\mathrm{mm}]}\end{array}$ & 6 & 5.6 & 6 & 4 \\
\hline $\begin{array}{c}\mathrm{H} \\
{[\mathrm{mm}]}\end{array}$ & 0 & 3.5 & 6 & 7 \\
\hline $\mathrm{n}$ & 6 & 6 & 6 & 10 \\
\hline
\end{tabular}

( $\mathrm{n}=$ number of tangential inlets)

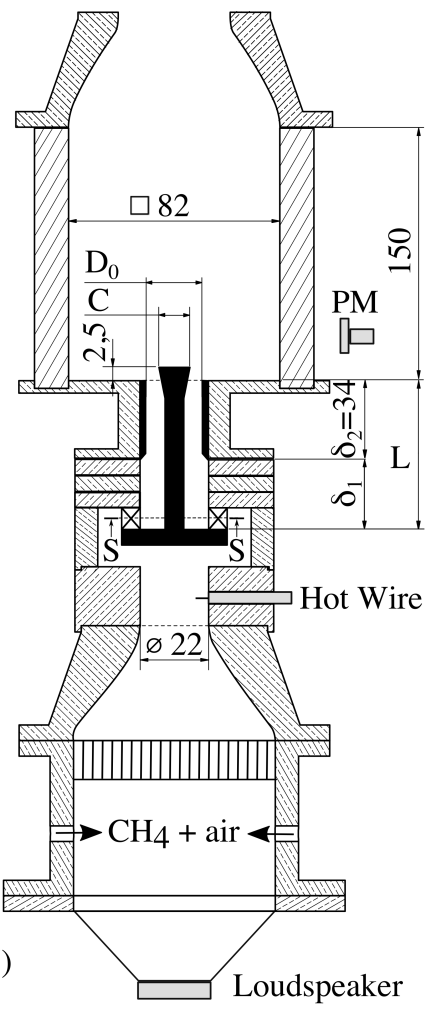

FIGURE 1: Burner sketch. The main dimensions are indicated in millimeters.

$20^{\circ} \mathrm{C}$ and $p=1 \mathrm{~atm}$ is deduced from the air and methane mass flowrates injected in the system and is kept constant for all the experiments conducted in this study. The system is thus operated at a constant thermal power $\mathscr{P}=5.44 \mathrm{~kW}$ assuming total combustion.

Four different radial swirlers with cylindrical tangential injection channels can be fixed in the injection unit to change the swirl level imparted to the flow. They are designated by $\mathrm{SW}_{i}$, with $i=1,2,3,4$ and their characteristics are synthesized in Fig. 1. The flow leaves the swirler into a central injection tube. The diameter of this tube is also $D=22 \mathrm{~mm}$, over a first section of length $\delta_{1}=22 \mathrm{~mm}$, followed by a nozzle of length $\delta_{2}=34 \mathrm{~mm}$ and a variable diameter $D_{0}=18,20$ or $22 \mathrm{~mm}$. A central rod of diameter $d=6 \mathrm{~mm}$, topped by a cone of variable diameter $C=10,12$ or $14 \mathrm{~mm}$ and length $10 \mathrm{~mm}$, is inserted inside the injection tube to ease flame stabilization. The cone protrudes $2.5 \mathrm{~mm}$ inside the combustion chamber. The distance between the swirler back-plane and the combustion chamber backplane is designated by $L=\delta_{1}+\delta_{2}=56 \mathrm{~mm}$.

The combustion chamber has a squared cross-section of $82 \mathrm{~mm}$ width and $150 \mathrm{~mm}$ length and is equipped with four quartz windows. At the base of the burner, a loudspeaker (Mona- 
cor SP-6/108PRO, 100 Watts RMS) is mounted to pulsate the flow. The velocity is measured with a hot wire anemometer probe (Dantec Dynamics - Probe 55P16 with a mini-CTA 54T30) below the swirler unit where the velocity has a top hat profile. A photomultiplier (Hamamatsu, H5784-04), equipped with a narrowband filter (Asahi Spectra, ZBPA310) centered around 310 $\mathrm{nm}$ is used to record the $\mathrm{OH}^{*}$ chemiluminescence signal. The hot wire and photomultiplier signals are used to determine the FTF.

A 2D-2C Particle Image Velocimetry (PIV) system is also used to analyze the flow structure at the injector outlet under steady and forced flow conditions. Small oil droplets of 1$3 \mu \mathrm{m}$ are used in this case to seed the flow and PIV measurements are limited to cold flow operation. The PIV system consists of $2 \times 400 \mathrm{~mJ} \mathrm{Nd}$ :YAG laser doubled at $532 \mathrm{~nm}$ operated at $10 \mathrm{~Hz}$ and a $2048 \times 2048 \mathrm{px}^{2} \mathrm{CCD}$ camera (Dantec Dynamics, FlowSense EO 4M). Two different optical setups are used with a time interval between the two laser pulses $\Delta t=10 \mu \mathrm{s}$ and a pixel pitch of $27.88 \mathrm{px} / \mathrm{mm}$ for measurements made in the axial plane and $\Delta t=25 \mu \mathrm{s}$ and a pixel pitch of $40.14 \mathrm{px} / \mathrm{mm}$ for measurements made in the transverse plane. Eight hundred images are taken to assure the convergence of the mean and rms values of the velocity field, which is computed from the cross-correlation of the PIV images by a three passes window deformation technique (from $64 \times 64 \mathrm{px}^{2}$ to $16 \times 16 \mathrm{px}^{2}$ interrogation areas), with an uncertainty of $0.1 \mathrm{px}$ on the calculated displacement.

An intensified CCD camera (Princeton Instruments, PIMAX 4, 1024×1024 $\mathrm{px}^{2}$ ), mounted with an UV objective (Nikkor $105 \mathrm{~mm} \mathrm{f/4.5)} \mathrm{and} \mathrm{equipped} \mathrm{with} \mathrm{the} \mathrm{same} \mathrm{filter} \mathrm{as} \mathrm{the}$ photomultiplier, is finally used to analyze the flame structure under steady and forced conditions. Phased averaged images of the $\mathrm{OH}^{*}$ signals and the PIV fields are synchronized by the signal driving the loudspeaker.

\section{STEADY INJECTION CONDITIONS}

PIV measurements under cold flow conditions are first used to compare the mean axial $\bar{u}_{z}$ and azimuthal $\bar{u}_{\theta}$ velocity components of the flow fields produced by the different injectors $2 \mathrm{~mm}$ above the top cone of the central bluff-body. Results obtained are shown in Fig. 2.

Swirler $\mathrm{SW}_{1}$ with the $D_{0}=22 \mathrm{~mm}$ nozzle diameter and the central cone $C=14 \mathrm{~mm}$ produces a typical axial velocity profile from a non-swirling flow in the wake of a bluff body. Due to small imperfections of rod centering and swirler manufacturing, the flow produced by $\mathrm{SW}_{1}$ featuring injection channels aligned with the radial direction of the reactant injection tube $(H=0 \mathrm{~mm}$, Fig. 1) features a small, but perceptible, azimuthal velocity component at the injector outlet. The residual azimuthal flow component remains weak, but could not be cancelled. Several PIV measurements were made to check this feature that was also found to be reproducible, with the same velocity profile, from tests to tests

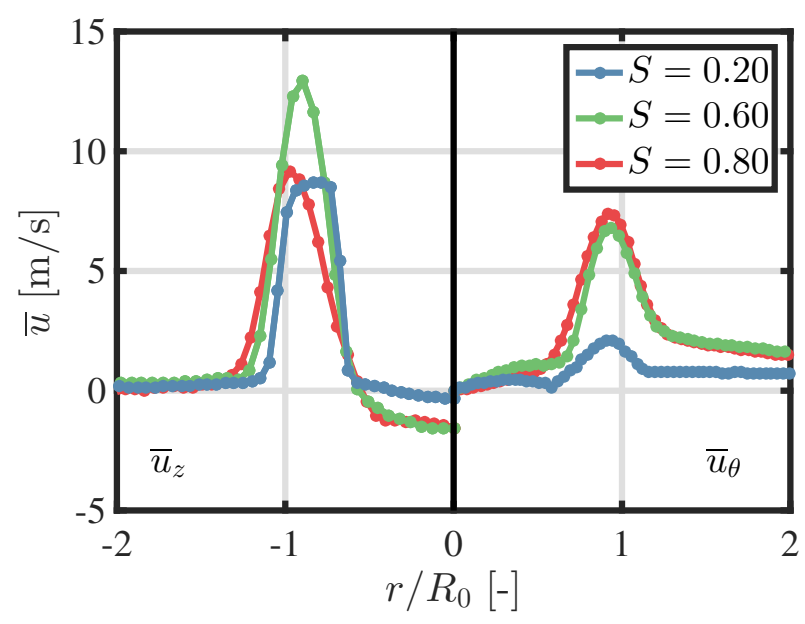

FIGURE 2: Mean axial $\bar{u}_{z}$ and azimuthal $\bar{u}_{\theta}$ velocity profiles produced by the different injectors and leading to different swirl numbers $S$ at the burner outlet. Blue: swirler $S W_{1}, D_{0}=22 \mathrm{~mm}$, $C=14 \mathrm{~mm}$. Green: swirler $S W_{3}, D_{0}=18 \mathrm{~mm}, C=12 \mathrm{~mm}$. Red: swirler $S W_{3}, D_{0}=20 \mathrm{~mm}, C=10 \mathrm{~mm}$.

by mounting and demounting the swirler and the rod. One may however note that this azimuthal component remains weak and the jet produced by this unit approaches that of a non-swirling jet. This velocity profile serves as a reference and is here compared to those obtained with swirler $\mathrm{SW}_{3}$.

Swirler $\mathrm{SW}_{3}$ is equipped with injection channels offset by $H=6 \mathrm{~mm}$ in Fig. 1 from the radial direction. This device now confers a large azimuthal velocity component to the flow in the combustion chamber. The resulting velocity profiles are shown in Fig. 2 for $\mathrm{SW}_{3}$ with two different nozzles and two different bluff bodies. Results in green correspond to those obtained with a nozzle diameter $D_{0}=18 \mathrm{~mm}$ and a top cone diameter $C=$ $12 \mathrm{~mm}$. Results in red are obtained for a nozzle diameter $D_{0}=$ $20 \mathrm{~mm}$ and a top cone diameter $C=10 \mathrm{~mm}$. The azimuthal flow component now takes high values in both cases, but remains roughly unaltered for these combinations of injection nozzle and top cone dimensions. The main differences between the flows produced by these injectors are seen in the axial velocity profiles. The nozzle with $D_{0}=18 \mathrm{~mm}$ and $C=12 \mathrm{~mm}$ has a reduced flow passage area compared to the one with $D_{0}=20 \mathrm{~mm}$ and $C=10 \mathrm{~mm}$, leading to much higher axial velocities.

These data are used to determine the swirl numbers at the injector outlet, which are calculated here as:

$$
S=\frac{2}{D_{0}} \frac{\int u_{\theta} u_{z} r^{2} d r}{\int u_{z}^{2} r d r}
$$

The values found for the swirl number are indicated in Fig. 2 with a relative precision $\pm 3 \%$ taking into account uncertainties on the 


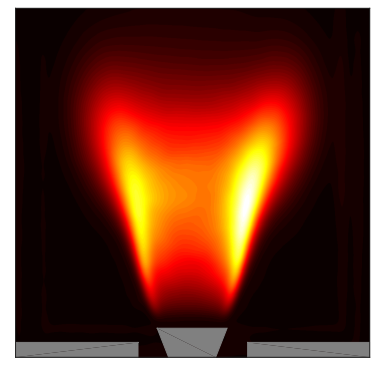

(a) $S=0.20$

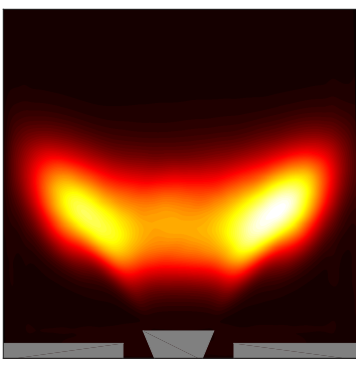

(b) $S=0.65$

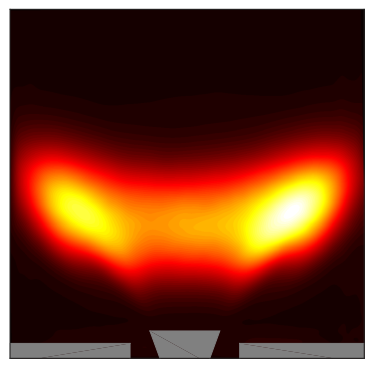

(c) $S=0.80$

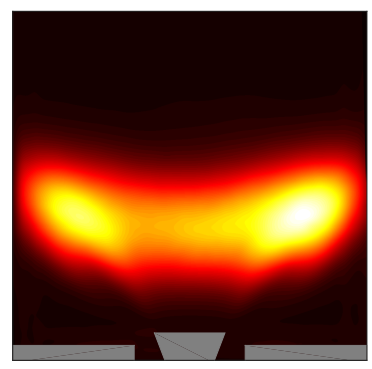

(d) $S=0.95$

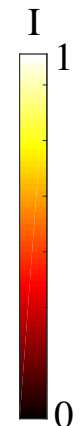

(a.u.)

FIGURE 3: $\mathrm{OH}^{*}$ light distribution for flames stabilized with the same injection nozzle diameter $D_{0}=22 \mathrm{~mm}$ and top cone central bluff body diameter $C=14 \mathrm{~mm}$, but different swirling vanes. (a) Swirler $\mathrm{SW}_{1}$. (b) Swirler $\mathrm{SW}_{2}$. (c) $\mathrm{Swirler} \mathrm{SW}_{3}$. (d) $\mathrm{Swirler} \mathrm{S} W_{4}$.

PIV measurements. For the other injectors tested in this study, the swirl number was deduced from geometrical considerations by assuming a uniform axial velocity profile and a linear profile for the azimuthal velocity component.

Effects of the swirl number $S$ on the shape taken by the flames are shown in Fig. 3. In these experiments, the injection nozzle diameter and the top cone of the central bluff body are fixed. It is also first worth recalling that the equivalence ratio $\phi=0.82$ and the bulk velocity $U_{b}=5.44 \mathrm{~m} / \mathrm{s}$ are the same. These images are obtained by averaging a hundred snapshots, each with a long exposure time of $20 \mathrm{~ms}$, that are recorded with the ICCD camera equipped with the $\mathrm{OH}^{*}$ filter. A small asymmetry is seen in the light distributions between the left and right part in these images. This is again due to small imperfections in machining and difficulties to center the bluff body over a relatively long distance from the swirler back plane, where it is screwed, to the injector outlet. The light distribution takes a relatively long and narrow shape concentrated in the wake of the central bluff-body for the low-swirling flame at $S=0.20$ obtained with swirler $\mathrm{SW}_{1}$ in Fig. 3a. The three other flames obtained for swirlers $S_{2}$, $\mathrm{SW}_{3}$ and $\mathrm{SW}_{4}$ spread over a much wider radial extension and are more compact in the axial direction. A closer look indicates that the height of the flame slightly reduces, while it spreads further in the radial direction as the swirl number increases from $S=0.65$ to $S=0.95$ in Figs. $3 b-d$.

These observations are well known features of swirling flames. More interestingly, the flame leading edge position remains roughly unaltered in Figs. 3b-d meaning that the flame lift-off distance remains constant, whereas the $\mathrm{OH}^{*}$ maximum light peaks slightly shift further away from the burner axis and toward the combustion chamber back plane as the swirl number increases from $S=0.65$ to $S=0.95$. It will be shown that these small differences have large consequences on the flame response to flowrate pulsations.

\section{FLAME TRANSFER FUNCTIONS}

The FTF is determined, for each of the four swirlers, from the velocity signal measured by the hot wire anemometer and the $\mathrm{OH}^{*}$ chemiluminescence signal measured by the photomultiplier gathering light from the whole combustion region and providing the mean $\bar{I}$ and fluctuating $I^{\prime}$ luminosity signals. Given that combustion is fully premixed and the system is operated at fuel lean conditions, the $\mathrm{OH}^{*}$ light signal is assumed to be a good tracer of the heat release rate. The loudspeaker, mounted at the bottom of the burner, provides the acoustic excitation in the frequency range $20-250 \mathrm{~Hz}$ and for a velocity fluctuation level $u^{\prime} / \bar{u}=0.30$ where $\bar{u}$ denotes the mean velocity component and $u^{\prime}$ the rootmean-square value (RMS) of the velocity fluctuation at the hot wire location. The cross- and power-spectral densities of the hot wire and photomultiplier signals evaluated at the forcing frequency $f$ are then used to determine the FTF :

$$
\mathscr{F}(f)=\frac{I^{\prime} / \bar{I}}{u^{\prime} / \bar{u}}=G(f) \exp (i \varphi(f))
$$

Results for increasing swirl levels at a fixed velocity perturbation level $u^{\prime} / \bar{u}=0.30 \mathrm{RMS}$ are shown in the left plot in Fig. 4. For the low swirl injector $S=0.20$ obtained with $\mathrm{SW}_{1}$, the FTF gain curve $G(f)$ smoothly increases to reach a maximum $G \simeq 1.27$ at $f=96 \mathrm{~Hz}$ and then smoothly decreases towards zero as the forcing frequency further increases. This low-pass filter behavior, with gain values exceeding unity in the frequency range $40-150 \mathrm{~Hz}$, is a well known feature of the acoustic response of laminar premixed non-swirling V-flames, as first theoretically demonstrated in [17] and confirmed experimentally in [18]. The same type of smooth behavior with large gain overshoots at low frequencies is also a characteristic of the response of turbulent non-swirling flames [19]. These reference data for $S=0.20$ are now used to examine effects of higher levels of swirl on the FTF obtained with different injector designs. 

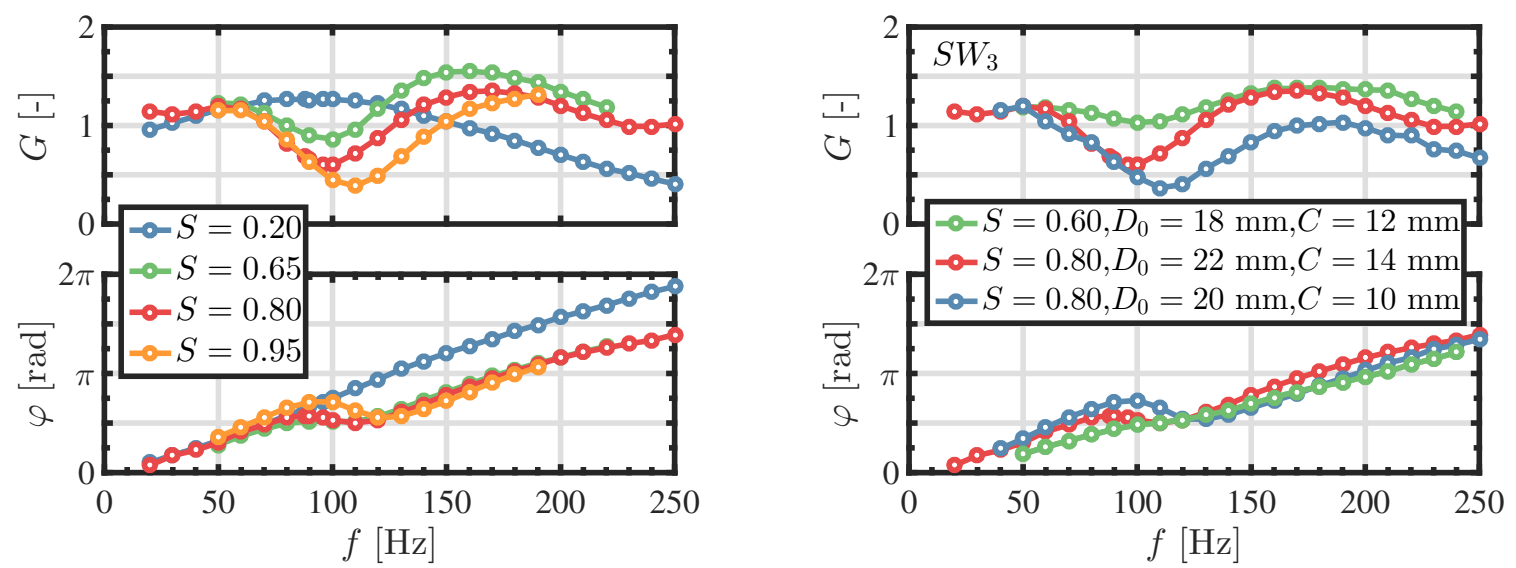

FIGURE 4: FTF at a fixed velocity perturbation level $u^{\prime} / \bar{u}=0.30$ RMS. Left: Effects of the swirl level $S$ for different injector designs. Blue: $\mathrm{SW}_{1}, D_{0}=22 \mathrm{~mm}, C=14 \mathrm{~mm}$. Green: $\mathrm{SW}_{2}, D_{0}=22 \mathrm{~mm}, C=14 \mathrm{~mm}$. Red: $\mathrm{SW}_{3}, D_{0}=22 \mathrm{~mm}, C=14 \mathrm{~mm}$. Orange: $\mathrm{SW}_{4}$, $D_{0}=22 \mathrm{~mm}, C=14 \mathrm{~mm}$. Right: Effects of the nozzle diameter and top cone diameter for a given swirling vane $\mathrm{SW}_{3}$.

The FTF of the swirled flames with $S \geq 0.65$ differ from that measured for $S=0.20$ by many aspects. The FTF gain curves of these flames in the top graph in Fig. 4 show a succession of a peak, a valley and another peak over a short frequency range, over which the FTF gain curve of the low swirled $S=0.20$ flame features a smooth behavior with large values exceeding unity. The highly swirled flames produced by the swirlers $\mathrm{SW}_{2}, \mathrm{SW}_{3}$ and $\mathrm{SW}_{4}$ reach their peak gain values at higher forcing frequencies with peak values slightly higher than the maximum gain value reached by the low swirled flame $S=0.20$. The frequency at which the lowest FTF gain value is observed weakly depends on the swirl number value for $0.65 \leq S \leq 0.95$.

Results are now presented in the right plot in Fig. 4 for the same swirling vane $\mathrm{SW}_{3}$, but for different values of the nozzle $D_{0}$ and bluff-body top cone $C$ diameters. The same features observed in the left figure are retrieved with a drop of the FTF gain curve in the intermediate frequency range that deepens as the swirl level increases from 0.60 (green curve) to 0.80 (red curve). One should however pay attention that the value of the swirl number $S$ is not the only parameter controlling the value of the FTF gain minimum. For a given swirl number $S$, adjusting the dimensions of the injector nozzle diameter $D_{0}$ and top cone diameter $C$ can be used to further reduce the minimum value of the FTF gain as highlighted by the red and blue curves obtained for $S=0.80$ in Fig. 4 .

Some of these features are now well understood and documented in a series of theoretical, numerical and experimental studies $[9-11,15,20]$. They are attributed to interferences between axial and azimuthal velocity fluctuations at the injector outlet. One results of the theoretical and experimental developments made in [11] is that the frequency $f_{0}$, at which the FTF gain of a swirling flame reaches its minimum, corresponds to a configuration where the flow at the burner outlet experiences the largest swirl number fluctuations, i.e. a situation where the azimuthal and axial velocity fluctuations are out of phase.

The frequency $f_{0}$ can be roughly estimated by considering the phase-lag $\varphi_{u_{\theta}^{\prime}-u_{z}^{\prime}}$ between azimuthal and axial velocity fluctuations at the combustor dump plane [10,12]:

$$
\varphi_{u_{\theta}^{\prime}-u_{z}^{\prime}}=2 \pi f_{0} \delta\left(\frac{1}{u_{c}}-\frac{1}{c}\right)
$$

where $u_{c}$ is the velocity at which azimuthal velocity fluctuations are convected and $c \gg u_{c}$ is the speed of sound. The condition for maximum swirl number oscillation, i.e. a minimum FTF gain, is reached when $\varphi_{u_{\theta}^{\prime}-u_{z}^{\prime}}=\pi$ modulo $2 \pi$. The distance $\delta$ is taken here equal to $50 \mathrm{~mm}$ and corresponds to the distance between the top of the swirler injection channels and the injector outlet. It is slightly smaller then the distance $L=56 \mathrm{~mm}$ from the bottom of the swirler back plate to the injector outlet. It was shown in $[11,12,21]$ that the velocity $u_{c}$ is close to the maximum velocity reached by the flow in the injection nozzle. This velocity remains unknown in the present study, but a rough estimate is made here by taking it close to the highest axial velocity measured at the injector outlet (see Fig. 2). The same choice was made in [11]. One finds $90 \leq f_{0} \leq 120 \mathrm{~Hz}$ for $9 \leq u_{c} \leq 12 \mathrm{~m} / \mathrm{s}$ and $\delta=50 \mathrm{~mm}$. The frequencies $f_{0}$ determined experimentally in Fig. 4 are within this range. The measured frequency $f_{0}=110 \mathrm{~Hz}$ is slightly higher in Fig. 4-left for device $\mathrm{SW}_{4}$ than the value $f_{0}=96 \mathrm{~Hz}$ found for the two other swirlers $\mathrm{SW}_{2}$ and $\mathrm{SW}_{3}$, despite the fact that the injector geometry is the same. This is attributed to the smaller diameter $D_{s}=4.0 \mathrm{~mm}$ of the holes from the swirling vane $\mathrm{SW}_{4}$ compared to the larger ones used for the devices $\mathrm{SW}_{2}$ and $\mathrm{SW}_{3}$. The smaller flow passage area 
for $\mathrm{SW}_{4}$ leads to a slightly higher velocity at the swirler channel outlets corresponding to a slightly higher frequency $f_{0}$ according to Eq. (3). To make a better prediction of the frequency $f_{0}$, one would need to know the exact value of the convection velocity $u_{c}$ of vortical disturbances over the distance $\delta$ from the swirling vane outlet to the injector outlet.

Striking features, which seem to have not been reported previously are observed when considering the FTF phase lag curves in the left plot in Fig. 4. The phase lag curves of the highly swirled flames obtained with the swirling vanes $\mathrm{SW}_{2}, \mathrm{SW}_{3}$ and $\mathrm{SW}_{4}$ feature the same evolution as the low swirled $S=0.20$ flame obtained with $\mathrm{SW}_{1}$ for frequencies $f<f_{0}$. This is not an obvious feature since the time lag $\tau$ appearing in the FTF phase lag $\varphi=2 \pi f \tau$ in Eq. (2) is generally considered to be proportional to the flame length $L_{f}$ and to the inverse of the bulk flow velocity $1 / U_{b}$. Figure 3 , obtained for steady injection conditions, clearly shows that the flame length $L_{f}$ and its aspect ratio largely differs between the flame stabilized with the device $\mathrm{SW}_{1}$ and the three other ones with $\mathrm{SW}_{2}, \mathrm{SW}_{3}$ and $\mathrm{SW}_{4}$.

At higher frequencies $f>f_{0}$, the FTF phase lag of the low swirled flame $S=0.20$ obtained with the vane $\mathrm{SW}_{1}$ keeps increasing in a regular fashion with an almost constant slope in the full frequency range investigated. An inflection point and a rapid change of the FTF phase lag characterize the responses of the highly swirled flames in the left plots in Fig. 4. At higher forcing frequencies the data for the FTF phase lags of the flames produced by the swirlers $\mathrm{SW}_{2}, \mathrm{SW}_{3}$ and $\mathrm{SW}_{4}$ roughly collapse on the same curve despite the differences in swirl numbers $S=0.65$, $S=0.80$ and $S=0.95$ respectively. The phase lag evolution at high frequencies is found to be roughly parallel, except at the highest forcing frequencies, to the FTF phase lag plot of the low swirled flame $S=0.20$ obtained with the device $\mathrm{SW}_{1}$ offset by a constant value of $\varphi_{0} \simeq-1.3 \mathrm{rad}$. A similar behavior is observed for the FTF phase lag curves measured for the same swirling vane $\mathrm{SW}_{3}$ but different nozzle and injector geometries in right plots in Fig. 4. One recalls that in all these experiments the bulk flow velocity $U_{b}$ and equivalence ratio $\phi$ remain unchanged. All the flames investigated are also stabilized in the wake of a central bluff body.

Another related feature is that the FTF gain of these flames obtained with different swirling vanes remains unaltered by the swirl level at very low forcing frequencies in the left and right plots in Figs. 4. Then the FTF gain decreases for forcing frequencies approaching the frequency $f_{0}$ where the FTF reaches a minimum. The minimum value reached by the FTF gain at $f_{0}$ regularly decreases as the swirl number $S$ increases. This was already observed in [15], where a limit of this FTF gain reduction has been identified as flashback takes place within the burner when the system is operated at a too large swirl number.

These observations can be used to guide further modeling of the FTF of swirling flames.

\section{COLD FLOW DYNAMICS}

Further analysis is now carried out under cold flow conditions by exploiting phase conditionned PIV measurements with the acoustic pulsation. Results are presented in Fig. 5. To identify vortical structures, the Q criterion is selected [22]:

$$
Q=\frac{1}{2}\left(|\Omega|^{2}-|\mathbf{S}|^{2}\right)
$$

where $\mathbf{S}$ and $\Omega$ are the symmetric and anti-symmetric components of the velocity gradient respectively. Iso-contours of $Q$ are inferred from velocity measurements conducted in the axial plane crossing the center of the combustion chamber. Only positive values of $Q$ are retained, while negative values, indicating areas of the flow where shear is present but no swirling motion, are forced to a zero value.

The first and third sequences in Fig. 5 correspond to forcing frequencies associated to a maximum of the FTF gain plots in Fig. 4. In both cases, a large vortical structure is generated at the rim of the injector in the external shear layer of the jet flow and is convected downstream in the chamber. This is highlighted by the high values taken by $Q$ in Fig. 5 where the trajectory of the structures is emphasized by the superposed dashed lines. In the second sequence in Fig. 5 corresponding to the forcing frequency $f_{0}=110 \mathrm{~Hz}$ associated to a minimum of the flame response in Fig. 4-Right, the large coherent structures shed from the burner lip can hardly be distinguished with low $Q$ values and are rapidly dissipated by the flow as they are convected.

A reduction of the strength of large vortical structures at the frequency corresponding to the FTF gain minimum was already observed in the analysis of the reacting flow dynamics of the swirled flames studied in $[14,23]$. In these studies, however, vortex damping was attributed to an earlier interaction between the flame dyamics and the vortical structures shed from the burner lip [14] and to damping of Kelvin-Helmholtz instabilities due their interactions with the heated flow [23]. From the analysis of Fig. 5, it appears that these vortical structures are damped even in the absence of heat release due to a purely hydrodynamic mechanism, which takes its origin from the complex interactions taking place between acoustic and vorticity waves inside the injector.

\section{FLAME DYNAMICS}

Phase averaged images of the flame $\mathrm{OH}^{*}$ chemiluminescence conditioned by the harmonic forcing excitation are examined in Fig. 6. The forcing level $u^{\prime} / \bar{u}=0.30 \mathrm{RMS}$ is the same as in Fig. 4 showing the FTF results. Six phase conditioned images covering the full cycle of oscillation are shown for the three configurations investigated. Each image, separated by a phase angle of 60 degrees, is formed by accumulating one hundred snapshots with an exposure of $40 \mu$ s each. The angles indicated in Fig. 6 

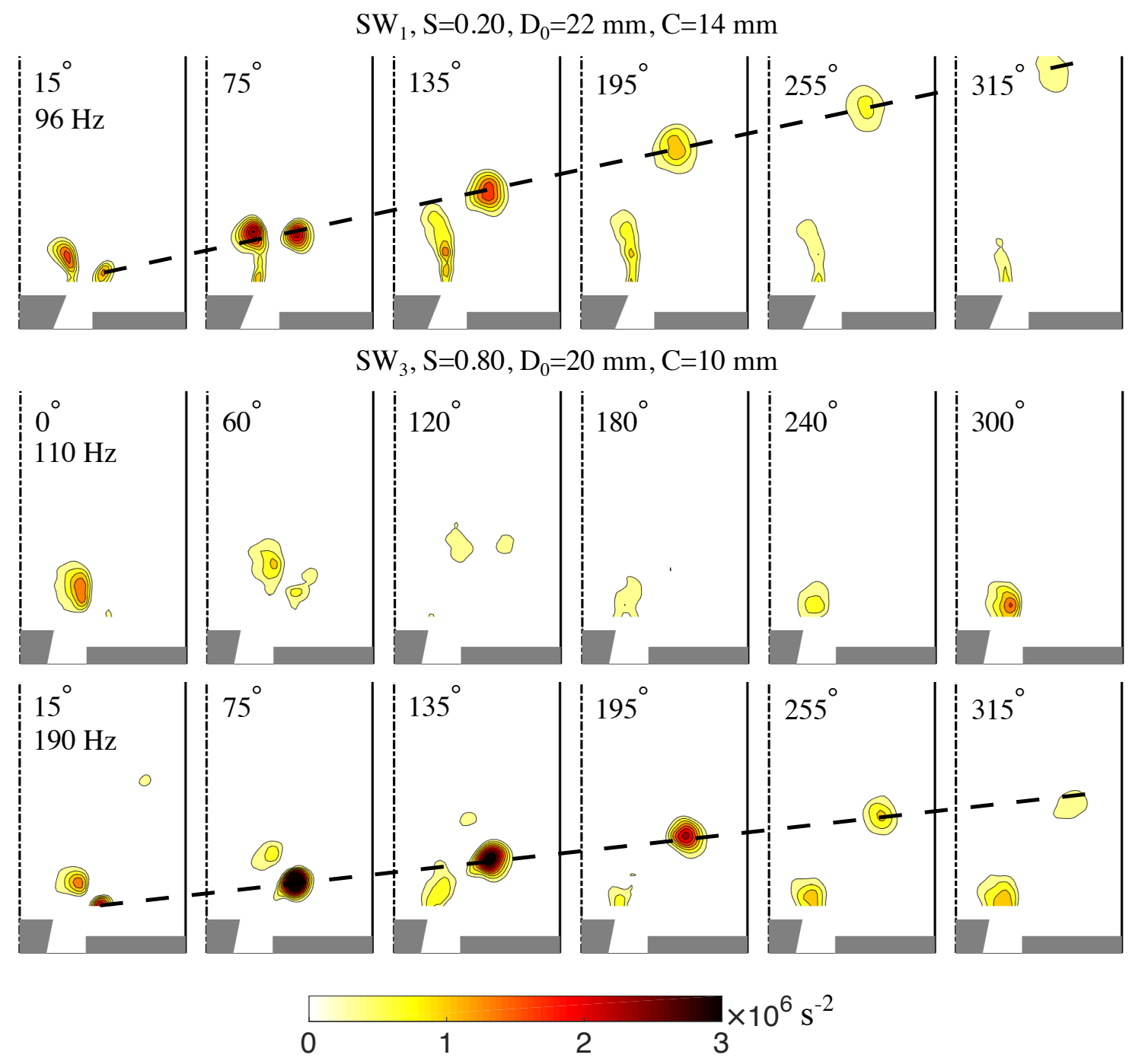

FIGURE 5: Q criterion contours deduced from PIV data in cold flow conditions for a forcing level $u^{\prime} / \bar{u}=0.30 \mathrm{RMS}$. The same colorbar, set from 0 to $3.10^{6} s^{-2}$, is applied to all figures. Only positive values of Q are retained. The phase angle is referred with respect to the velocity signal measured by the hot wire probe.

are the phase delays with respect to the hot wire probe signal. An Abel deconvolution reveals the trace of the flame luminosity in the axial plane crossing the burner axis. The same color scale is used for all images to better highlight both the flame motion and changes of the flame luminosity during the forcing cycle. The flame contours, highlighting the region of the flame where the luminosity is higher than a threshold level, is also shown. The threshold is taken here equal to $25 \%$ of the maximum flame luminosity in each phase averaged picture, a value found to be suitable for the determination of the flame contours. It was checked that the results are only weakly sensitive to this threshold level, when it is chosen in the range $15-35 \%$ of the maximum flame luminosity.

The first sequence in Fig. 6 highlights the large motion executed by the flame stabilized in the low swirl $S=0.20$ flow produced by the device $\mathrm{SW}_{1}$ when excited at the frequency $f=96 \mathrm{~Hz}$ corresponding to the FTF gain maximum in Fig. 4Left. Large roll-up of the flame tip is seen at the phases $195^{\circ}$ and $255^{\circ}$. The flame is strongly stretched in the vertical direction during the forcing cycle with relatively minor changes of the $\mathrm{OH}^{*}$ luminosity.

The second and third sequences in Fig. 6 show the flame responses for a higher swirl number $S=0.80$ produced with the swirler $\mathrm{SW}_{3}$. The images are gathered at the forcing frequen- 
$\mathrm{SW}_{1}, \mathrm{~S}=0.20, \mathrm{D}_{0}=22 \mathrm{~mm}, \mathrm{C}=14 \mathrm{~mm}$
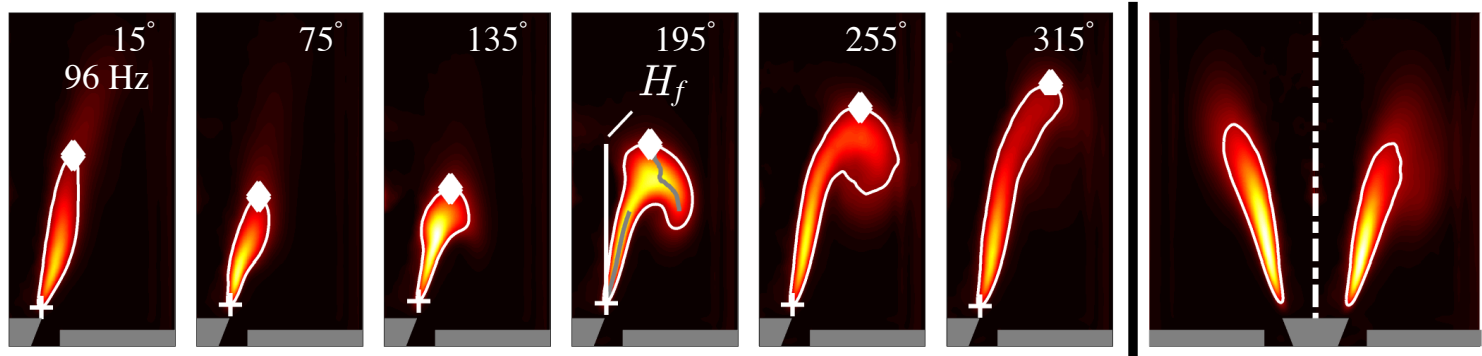

$\mathrm{SW}_{3}, \mathrm{~S}=0.80, \mathrm{D}_{0}=20 \mathrm{~mm}, \mathrm{C}=10 \mathrm{~mm}$
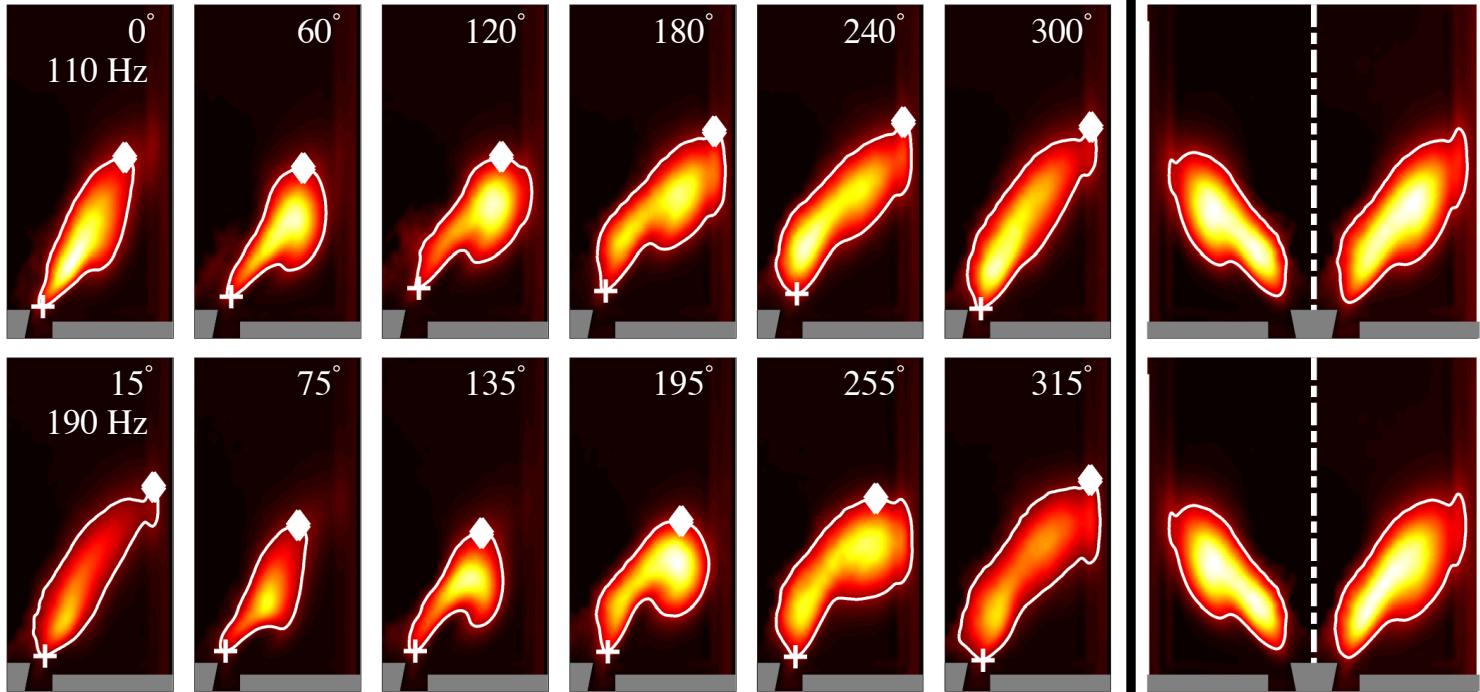

(a)
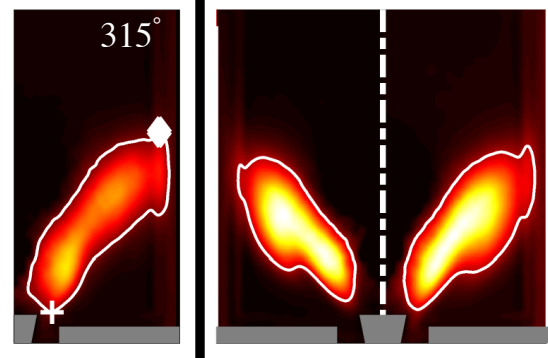

(b)

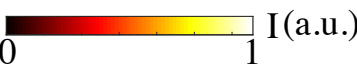

FIGURE 6: (a): Abel deconvoluted phase conditioned images of the flame $\mathrm{OH}^{*}$ signal for a forcing level $u^{\prime} / \bar{u}=0.30$ RMS. The phase angle is referred to the hot wire signal. The average flame position used to determine the flame height $H_{f}$ and flame base angle $\alpha$ is shown in the first row at the phase angle $195^{\circ}$. (b): comparison between unforced flame (left of the chamber axis) and the mean flame determined from the forced phase averaged images (right of the chamber axis).

cies $f_{0}=110 \mathrm{~Hz}$ corresponding to the FTF gain minimum and $f=190 \mathrm{~Hz}$ corresponding to the FTF gain maximum in Fig. 4Right. The motion experienced by the flame at these two forcing frequencies does not differ significantly, but it is mainly changes of the flame luminosity that explain the large differences observed for the FTF gain at $f_{0}=110 \mathrm{~Hz}$ and $f=190 \mathrm{~Hz}$ in Fig. 4 . At $f_{0}=110 \mathrm{~Hz}$, there is a relatively weak flame roll-up motion in Fig. 6 accompanied by weak changes of the flame luminosity over the phases of the forcing cycle. At $f=190 \mathrm{~Hz}$, the flame roll-up motion is a bit further pronounced, but the $\mathrm{OH}^{*}$ luminosity undergoes large regular changes over the phases in the forcing cycle explaining the large value taken by the FTF gain at this frequency in Fig. 4.

In the last column in Fig. 6, the $\mathrm{OH}^{*}$ light distributions from the unperturbed flames are compared to the mean flame light distributions averaged over all phase conditioned images in the row. One clearly sees that this average distribution remains unaltered at the forcing frequencies characterizing the minimum and maximum flame responses. In all configurations explored, this average light distribution also barely differs from the unperturbed flame light distribution even for a forcing level as high as $u^{\prime} / \bar{u}=0.30$ RMS. The main differences between the unperturbed images and average images of the perturbed distributions are (i) a small decrease of the mean flame lift-off distance, (ii) a small modification of the flame height and (iii) an increased flame volume in the region where vortical structures impinge the flame.

Images in Fig. 6 are now used to determine the average flame 


$$
\multimap \frac{\mathrm{H}_{\mathrm{f}}^{\prime}}{\overline{\mathrm{H}_{\mathrm{f}}}} \multimap \frac{\alpha^{\prime}}{\bar{\alpha}} \multimap \mathrm{S}
$$
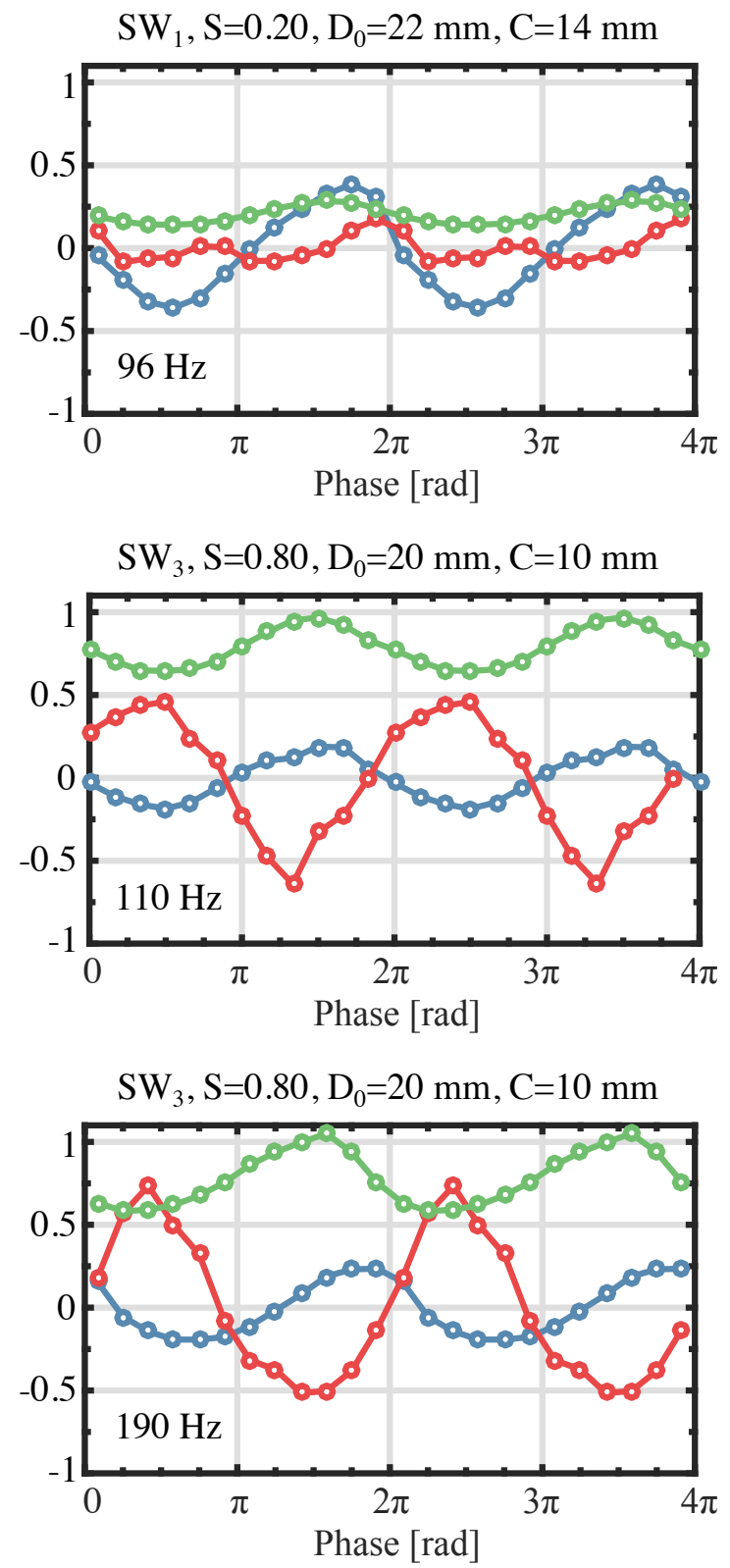

FIGURE 7: Evolution of the swirl number $S$ (green curve), flame height normalized fluctuation $H_{f}^{\prime} / \overline{H_{f}}$ (blue curve) and flame angle normalized fluctuation $\alpha^{\prime} / \bar{\alpha}$ at the flame base (red curve). The phase is referred to the hot-wire signal. Two cycles are represented for better readability.

position in each image recorded. This position is superimposed in grey on the $195^{\circ}$ flame image in the first sequence in Fig. 6.
This profile is obtained by finding the maximum row-wise intensity of the pixel, weighted by the distance from the burner axis as expressed by: $\int I(r, x) 2 \pi r d r$. Once the average flame position is determined for each phase-conditioned picture, the flame height $H_{f}$ is deduced by calculating the length of the vertical white line, i.e. the vertical distance between the lowest (shown as a plus sign in Fig. 6) and the highest (shown as a diamond sign in Fig. 6) points of the average flame light distribution as exemplified in the first row in Fig. 6 at $195^{\circ}$. This process proves to be efficient even when the flame is strongly modulated by vortex rollup in Fig. 6. The flame angle $\alpha$ with respect to the vertical direction is also determined at the flame leading edge position. Finally, the evolution of the swirl number $S$ is also determined, for each selected phase in the cycle, using the PIV data in a longitudinal plane crossing the chamber axis and in a transverse plane $2 \mathrm{~mm}$ above the top cone of the central bluff-body, with a relative precision close to $\pm 5 \%$. Results for the flame height $H_{f}$ and flame base angle $\alpha$ are plotted over two periods in Fig. 7 together with the corresponding swirl number in the forcing cycle.

In the top graph in Fig. 7, obtained for the device $\mathrm{SW}_{1}$ and a low swirl number $S=0.20$, the flame undergoes large fluctuations of its height $H_{f}^{\prime} / \bar{H}_{f}$, but no oscillation of the flame base angle $\alpha^{\prime} / \bar{\alpha} \simeq 0$. The small oscillations of the swirl number in this plot are not significant here due to the low values of the swirl number $S \simeq 0.2$. The situation differs for the results obtained with swirler $\mathrm{SW}_{3}$ for a swirl number $S=0.80$ in the middle and bottom graphs in Fig. 7. The flame responses now exhibit large flame base angle $\alpha^{\prime} / \bar{\alpha}$, flame height $H_{f}^{\prime} / \bar{H}_{f}$ and swirl number oscillations $S^{\prime} / \bar{S}$ at the two forcing frequencies. The main striking features are that the swirl number, the flame base angle and the flame height oscillations experienced by these flames are very similar at $f=110 \mathrm{~Hz}$ and $f=190 \mathrm{~Hz}$. Synchronization between these different signals is also roughly the same. This confirms that there are no significant differences between the motions experienced by these flames when they are forced at a frequency $f$ corresponding to a minimum in the FTF gain curve $(110 \mathrm{~Hz})$ or a maximum $(190 \mathrm{~Hz})$. Another important feature is that large swirl number oscillations are detected for both flames featuring low but also large FTF gain values. These two last observations contrast with current interpretations of the dynamics controlling the flame responses to swirl level oscillations [14,23].

A deeper insight into the mechanisms leading to low or high FTF gain values is attempted by decomposing heat release rate fluctuations $\dot{Q}^{\prime} / \bar{Q}$ as the sum of fluctuations associated to changes of the volumetric rate of heat release averaged over the flame volume $<\dot{q}^{\prime}>/<\bar{q}>$ and fluctuations associated to the flame volume $V^{\prime} / \bar{V}$ with the methodology described in [24]:

$$
\frac{\dot{Q}^{\prime}}{\dot{\bar{Q}}}=\frac{<\dot{q}^{\prime}>}{<\dot{q}>}+\frac{V^{\prime}}{\bar{V}}
$$



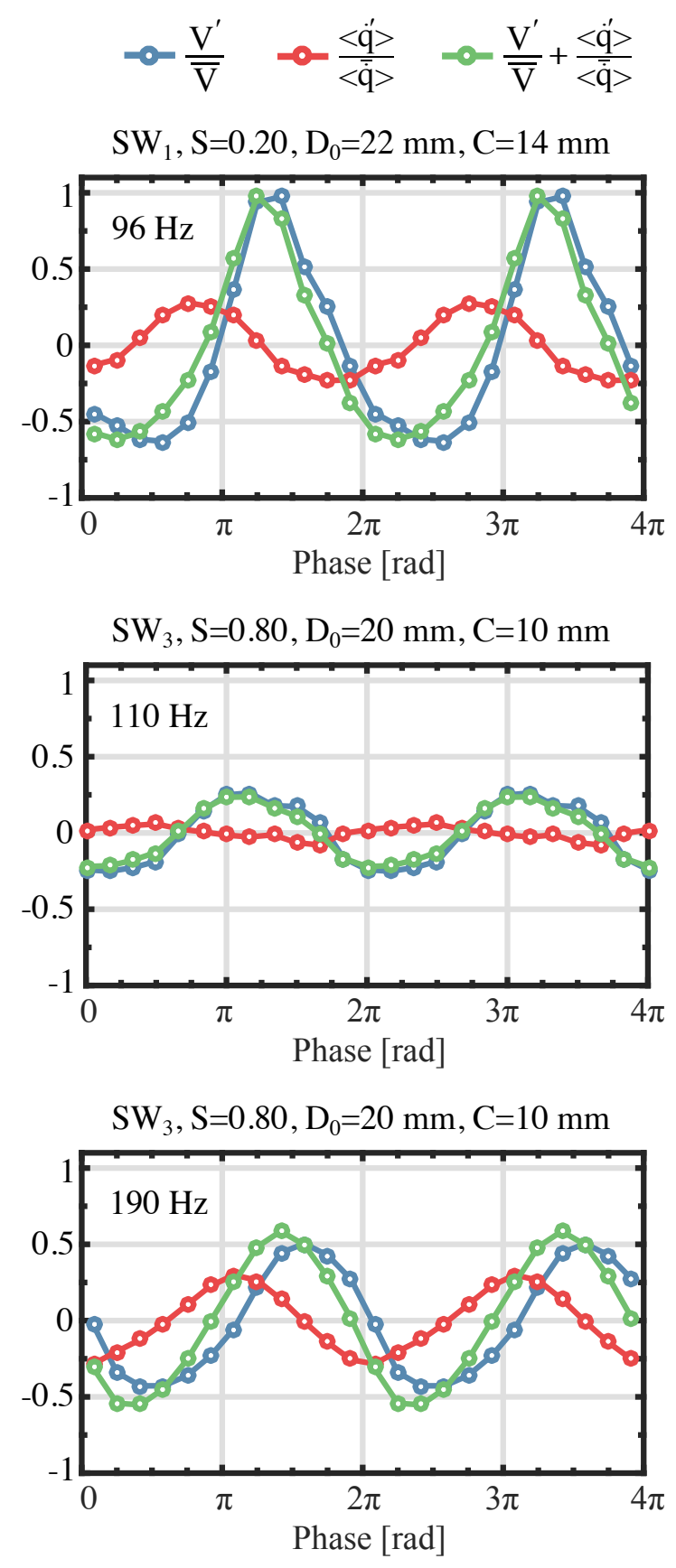

FIGURE 8: Evolution of the spatially-averaged volumetric heat release rate fluctuation $\left.\left\langle\dot{q}^{\prime}\right\rangle /<\overline{\dot{q}}\right\rangle$ (red curve), flame volume fluctuation $V^{\prime} / \bar{V}$ (blue curve) and sum of these contributions (green curve). The phase is referred to the hot-wire signal. Two cycles are represented for better readability.

The evolution of these three quantities is shown in Fig. 8 for the three cases explored in Figs. 6 and 7. At the low swirl number $S=0.20$, heat release rate oscillations $\dot{Q}^{\prime} / \overline{\dot{Q}} \simeq V^{\prime} / \bar{V}$ are mainly driven by changes of the flame volume with only a slight contribution from local changes of the volumetric heat release rate $\left\langle\dot{q}^{\prime}>/<\bar{q}>\right.$ in the top row in Fig. 8. In this case, the flame luminosity remains roughly constant over the forcing cycle in Fig. 6.

For the flame stabilized with the swirler $\mathrm{SW}_{3}$ and the swirl number $S=0.80$ which is excited at $f_{0}=110 \mathrm{~Hz}$, the $<\dot{q}^{\prime}>/<$ $\overline{\dot{q}}>\simeq 0$ almost vanishes in the middle graph in Fig. 8 and $V^{\prime} / \bar{V}$ largely drops compared to its fluctuation in the top graph. This analysis confirms that at the frequency corresponding to a minimum FTF gain, fluctuations of the volumetric heat release rate and flame volume remain weak. This corroborates the absence of large structures impinging the flame as observed in the analysis of the perturbed cold flow at the same forcing frequency.

In the last graph in Fig. 8 obtained for a forcing frequency $f=190 \mathrm{~Hz}$, and corresponding to a maximum value for the FTF gain, the contributions from $V^{\prime} / \bar{V}$ and $<\dot{q}^{\prime}>/<\overline{\dot{q}}>$ reach both comparable and relatively high levels. This means that large vortical structures impinging the flame modulate both the flame shape but also the local consumption rate.

This analysis confirms the qualitative descriptions made in the discussion of Fig. 6. At the frequencies corresponding to the lowest flame responses, (i) the flame luminosity barely changes during the forcing cycle and (ii) the flame may eventually execute a motion during the forcing cycle, but its surface variation remains low. These two mechanisms explain the low FTF responses observed in this work and confirm that low heat release rate oscillations observed at specific frequencies of the swirled flames investigated are not associated here with a compensation mechanism between out of phase contributions to heat release rate oscillations originating from different regions of the flames. Low heat release rate fluctuations results here from the absence of large vortical structures shed from the burner lip, that are also absent in the cold flow response at the same excitation frequency.

\section{CONCLUSION}

The acoustic response of flames stabilized with increasing values of the swirl number has been investigated with a series of experiments. It is confirmed that the FTF gain curve of swirling flames is characterized by valleys and peaks in the low frequency range. It is also confirmed that the minimum gain value decreases as the swirl number increases. This behavior is the consequence of interferences between the acoustic pulsation and azimuthal flow disturbances generated at the swirler outlet. It is shown that this interaction depends on the swirl number and the excitation frequency.

The mechanisms leading to high or low responses of swirling flames have been investigated by PIV measurements of the cold flow response to the acoustic excitation. At frequencies corresponding to FTF gain maxima, it has been found that 
large coherent vortical structures are convected in the external shear layer of the flow and dominate the flow response. At frequencies corresponding to FTF gain mimima, these structures are rapidly damped in the cold flow. This leads to the conclusion that the low response of swirling flames observed at specific forcing frequency is a purely hydrodynamic mechanism taking place between azimuthal vortical disturbances and axial flow disturbances.

A phase conditioned analysis of the flame responses to acoustic forcing reveals that, when the FTF gain is at a maximum, the flame is strongly modulated by the interaction with the large vortical structures identified in the cold flow response. When the swirl number is low, this interaction leads to a strong modulation of the flame height during the forcing cycle, while the flame base angle is not altered. Heat release rate oscillations mainly result in this case from oscillations of the flame volume. When the swirl number is high, the flame base angle and the flame height are both modulated by the incoming flow disturbances. The heat release rate oscillation results in this case from large changes of the volumetric rate of heat released and of the flame volume. For conditions corresponding to a FTF gain minimum, fluctuations of the volumetric rate of heat released are drastically reduced and only small fluctuations of the flame volume are observed. These reductions are associated to a strong weakening of the large vortical structures shed from the burner as observed in the cold flow response. The experiments made in this work reveal a purely hydrodynamic mechanism at the origin of the low response of swirling flames at certain specific frequencies.

\section{ACKNOWLEDGMENTS}

This project has received funding from the European Union's Horizon 2020 research and innovation programme under the Marie Sklodowska-Curie grant agreement No 643134. This work is supported by Agence Nationale de la Recherche, NOISEDYN project (ANR-14-CE35-0025-01). The authors are thankful to the technical staff of EM2C laboratory for their help.

\section{REFERENCES}

[1] Keller, J. J., 1995. "Thermoacoustic oscillations in combustion chambers of gas turbines". AIAA J., 33(12), pp. 22802287.

[2] Zinn, B. T., and Lieuwen, T. C., 2005. "Combustion Instabilities: Basic Concepts". In Combustion Instabilities in Gas Turbine Engines, T. C. Lieuwen and V. Yang, eds. pp. 3-24.

[3] Poinsot, T., 2017. "Prediction and control of combustion instabilities in real engines". Proc. Combust. Inst., 36(1), pp. 1-28.
[4] Syred, N., 2006. "A review of oscillation mechanisms and the role of the precessing vortex core (PVC) in swirl combustion systems". Prog. Energy Combust. Sci., 32, pp. 93161.

[5] Huang, Y., and Yang, V., 2009. "Dynamics and stability of lean-premixed swirl-stabilized combustion". Prog. Energy Combust. Sci., 35(4), pp. 293-364.

[6] Candel, S., Durox, D., Schuller, T., Bourgouin, J.-F., and Moeck, J. P., 2014. "Dynamics of Swirling Flames". Annu. Rev. Fluid Mech., 46, pp. 147-173.

[7] Wang, S., Hsieh, S. Y., and Yang, V., 2005. "Unsteady flow evolution in swirl injector with radial entry. I. Stationary conditions". Phys. Fluids, 17(4), p. 045106.

[8] Straub, D. L., and Richards, G. A., 1999. "Effect of axial swirl vane location on combustion dynamics". In ASME Pap. 99-GT-109.

[9] Komarek, T., and Polifke, W., 2010. "Impact of Swirl Fluctuations on the Flame Response of a Perfectly Premixed Swirl Burner". J. Eng. Gas Turbines Power, 132(6), p. 061503.

[10] Kim, K. T., and Santavicca, D. A., 2013. "Interference mechanisms of acoustic / convective disturbances in a swirl-stabilized lean-premixed combustor". Combust. Flame, 160(8), pp. 1441-1457.

[11] Palies, P., Durox, D., Schuller, T., and Candel, S., 2010. "The combined dynamics of swirler and turbulent premixed swirling flames”. Combust. Flame, 157(9), pp. 1698-1717.

[12] Palies, P., Durox, D., Schuller, T., and Candel, S., 2011. "Acoustic - convective mode conversion in an aerofoil cascade". J. Fluid Mech., 672, pp. 545-569.

[13] Palies, P., Durox, D., Schuller, T., and Candel, S., 2011. "Experimental study on the effect of swirler geometry and swirl number on flame describing functions". Combust. Sci. Technol., 183(7), pp. 704-717.

[14] Palies, P., Schuller, T., Durox, D., Gicquel, L. Y., and Candel, S., 2011. "Acoustically perturbed turbulent premixed swirling flames". Phys. Fluids, 23(3), p. 037101.

[15] Gatti, M., Gaudron, R., Mirat, C., and Schuller, T., 2017. "Effects of the injector design on the transfer function of premixed swirling flames". In Proc. ASME Turbo Expo. GT2017-63874.

[16] Gatti, M., Gaudron, R., Mirat, C., and Schuller, T., 2017. "Injection System Design Impact on the Stabilization and Acoustic Response of Premixed Swirling Flames". Eur. Combust. Meet. ECM2017, pp. 1-6.

[17] Schuller, T., Durox, D., and Candel, S., 2003. "A unified model for the prediction of laminar flame transfer functions: comparisons between conical and V-flame dynamics". Combust. Flame, 134(1-2), pp. 21-34.

[18] Durox, D., Schuller, T., and Candel, S., 2005. "Combustion dynamics of inverted conical flames". Proc. Combust. Inst., 30(2), pp. 1717-1724. 
[19] Balachandran, R., Ayoola, B. O., Kaminski, C. F., Dowling, A. P., and Mastorakos, E., 2005. "Experimental investigation of the nonlinear response of turbulent premixed flames to imposed inlet velocity oscillations". Combust. Flame, 143, pp. 37-55.

[20] Palies, P., Schuller, T., Durox, D., and Candel, S., 2011. "Modeling of premixed swirling flames transfer functions". Proc. Combust. Inst., 33(2), pp. 2967-2974.

[21] Acharya, V., and Lieuwen, T., 2015. "Effect of azimuthal flow fluctuations on flow and flame dynamics of axisymmetric swirling flames". Phys. Fluids, 27(10), p. 105106.

[22] Jeong, J., and Hussain, F., 1995. "On the identification of a vortex". J. Fluid Mech., 285, pp. 69-94.

[23] Bunce, N. A., Quay, B. D., and Santavicca, D. A., 2013. "Interaction Between Swirl Number Fluctuations and Vortex Shedding in a Single-Nozzle Turbulent Swirling FullyPremixed Combustor". J. Eng. Gas Turbines Power, 136(2), p. 021503.

[24] Gaudron, R., Gatti, M., Mirat, C., and Schuller, T., 2017. "Impact of the injector size on the transfer functions of premixed laminar conical flames". Combust. Flame, 179, pp. 138-153. 